\title{
A Double Inequality for the Harmonic Number in Terms of the Hyperbolic Cosine
}

\author{
Da-Wei Niu ${ }^{1, *}$, Yue-Jin Zhang ${ }^{1}$, Feng $\mathbf{Q i}^{2,3,4}$ \\ ${ }^{1}$ College of Information and Business, Zhongyuan University of Technology, Zhengzhou City, Henan Province, China \\ ${ }^{2}$ College of Mathematics, Inner Mongolia University for Nationalities, Tongliao City, Inner Mongolia Autonomous Region, China \\ ${ }^{3}$ Department of Mathematics, College of Science, Tianjin Polytechnic University, Tianjin City, China \\ ${ }^{4}$ Institute of Mathematics, Henan Polytechnic University, Jiaozuo City, Henan Province, China \\ *Corresponding author: nnddww@163.com
}

Received November 03, 2014; Revised December 03, 2014; Accepted December 11, 2014

\begin{abstract}
In the paper, the author present an inequality for bounding the harmonic number in terms of the hyperbolic cosine.
\end{abstract}

Keywords: Inequality, Euler-Mascheroni constant, Harmonic number, Hyperbolic cosine

Cite This Article: Da-Wei Niu, Yue-Jin Zhang, Feng Qi, and Feng Qi, "A Double Inequality for the Harmonic Number in Terms of the Hyperbolic Cosine." Turkish Journal of Analysis and Number Theory, vol. 2, no. 6 (2014): 223-225. doi: 10.12691/tjant-2-6-6.

\section{Introduction}

The harmonic number $H_{n}$ is defined as

$$
H_{n}=1+\frac{1}{2}+\frac{1}{3}+\cdots+\frac{1}{n}
$$

and it has the following close connections with the EulerMascheroni constant $\gamma$ :

$$
\gamma=\lim _{n \rightarrow \infty}\left(H_{n}-\ln n\right)=0.57721 \ldots
$$

and

$$
H_{n}=\psi(n+1)+\gamma
$$

where $\psi(x)$ is the digamma function which is the logarithmic derivative of the classical Euler gamma function

$$
\Gamma(z)=\int_{0}^{\infty} t^{z-1} e^{-t} d t, \mathfrak{R}(z)>0 .
$$

The harmonic number $H_{n}$ has interesting applications in many areas of mathematics, such as number theory, special functions, and combinatorics. For example, Lagarias proved that the Riemann hypothesis is equivalent to the statement that

$$
\sigma(n) \leq H_{n}+e^{H_{n}} \ln H_{n}
$$

for $\mathrm{n} \in \mathbb{N}$, where $\sigma(n)$ denotes the sum of the divisors of n.

In [20], Paule and Schneider obtained the identity

$$
\sum_{k=0}^{n}\left(\begin{array}{l}
k \\
n
\end{array}\right)^{2} H_{n}=\left(\begin{array}{c}
n \\
2 n
\end{array}\right)\left(2 H_{n}-H_{2 n}\right) .
$$

In [2], Alzer presented the inequality

$$
\alpha \frac{\ln (\ln n+\gamma)}{n^{2}} \leq H_{n}^{1 / n}-H_{n+1}^{1 /(n+1)}<\beta \frac{\ln (\ln n+\gamma)}{n^{2}}
$$

for $\mathrm{n} \geq 2$, where the constants $\alpha=0.0140 \ldots$ and $\beta=1$ are the best possible. In [5], Batir gave an inequality

$$
\ln \frac{\pi^{2}}{6}-\ln \left(e^{1 /(n+1)}-1\right)<H_{n}<\gamma-\ln \left(e^{1 /(n+1)}-1\right) .
$$

This double inequality was refined in [4] by replacing $\ln \frac{\pi^{2}}{6}$ by 1 . It also inspired Mortici to construct a sequence

$$
\mu_{n}=\sum_{k=1}^{n} \frac{1}{k}+\ln \left(e^{a /(n+b)}-1\right)-\ln a
$$

in [15], which converges to more quickly.

For more information on the harmonic number $H_{n}$, please refer to $[2,6-19,21-26]$ and plenty of references therein.

In this paper, we will establish a new double inequality for bounding the harmonic number $H_{n}$ in terms of the hyperbolic cosine.

Our main result may be stated as the following theorem.

Theorem 1.1. For all positive integers $n \in \mathbb{N}$, we have

$$
\alpha \leq H_{n}-\ln n-\ln \cosh \frac{1}{\sqrt{n}}<\beta,
$$

where the constants $\alpha=1-\ln (\cosh 1)=0.5662 \ldots$ and $\beta=\gamma=0.5772 \ldots$ are the best impossible.

\section{Lemmas}

In order to prove Theorem 1.1, we need the following lemmas. 
Lemma 2.1 ([[3], p. 384]). Let $n \geq 1$ and $k \geq 0$ be integers, for $\mathrm{x}>0$, we have

$$
S_{n}(2 k ; x)<(-1)^{(n+1)} \psi^{(n)}(x)<S_{n}(2 k+1 ; x),
$$

where

$$
S_{n}(k ; x)=\frac{(n-1) !}{x^{n}}+\frac{n !}{2 x^{n+1}}+\sum_{i=1}^{k} \frac{B_{2 j}}{x^{2 i+n}} \prod_{j=1}^{n-1}(2 i+j) .
$$

Lemma $2.2([10,22])$. For $x>0$, we have

$$
\psi^{\prime}(x+1)>\frac{1}{x}-\frac{1}{2 x^{2}}+\frac{1}{6 x^{3}}-\frac{1}{30 x^{5}} .
$$

\section{Proof of Theorem 1.1}

Now we are in a position to prove our Theorem 1.1. Let

$$
f(x)=\psi(x+1)-\ln x-\ln \cosh \frac{1}{\sqrt{x}}+\gamma, x>0 .
$$

A direct differentiation yields

$$
f^{\prime}(x)=\psi^{\prime}(x+1)-\frac{1}{x}-\frac{e^{2 / \sqrt{x}}-1}{2\left(1+e^{2 / \sqrt{x}}\right) x^{3 / 2}}
$$

and

$$
\begin{aligned}
& 2 f^{\prime}(x)\left(1+e^{2 / \sqrt{x}}\right) x^{3 / 2}=2\left(1+e^{2 / \sqrt{x}}\right) x^{3 / 2} \psi^{\prime}(x+1) \\
& -1+(1-2 \sqrt{x}) e^{2 / \sqrt{x}}-2 \sqrt{x} \\
& =\left(1+e^{2 / \sqrt{x}}\right)\left[1-2 \sqrt{x}+2 x^{3 / 2} \psi^{\prime}(x+1)\right]-2 \triangleq g(x) .
\end{aligned}
$$

By virtue of inequalities (2.1) and

$$
e^{2 / \sqrt{x}}>1+\frac{2}{\sqrt{x}}+\frac{2}{x}+\frac{4}{3 x \sqrt{x}}+\frac{2}{3 x^{2}}+\frac{4}{15 x^{2} \sqrt{x}}+\frac{4}{45 x^{3}}
$$

we acquire

$$
\begin{aligned}
g(x)> & \left(2+\frac{2}{\sqrt{x}}+\frac{2}{x}+\frac{4}{3 x \sqrt{x}}+\frac{2}{3 x^{2}}\right. \\
& \left.+\frac{4}{15 x^{2} \sqrt{x}}+\frac{4}{45 x^{3}}\right)[1-2 \sqrt{x} \\
& \left.+2 x^{2 / 3}\left(\frac{1}{x}-\frac{1}{2 x^{2}}+\frac{1}{6 x^{3}}-\frac{1}{30 x^{5}}\right)\right]-2 \\
= & \frac{2}{675 x^{13 / 2}}\left(-2-6 x^{1 / 2}-15 x-30 x^{3 / 2}\right. \\
- & \left.35 x^{2}-15 x^{5 / 2}+90 x^{7 / 2}+90 x^{4}\right) \\
> & \frac{2}{675 x^{13 / 2}}\left(-2-6 x-15 x-30 x^{2}\right. \\
- & \left.35 x^{2}-15 x^{3}+90 x^{3}+90 x^{4}\right) \\
= & \frac{1}{675 x^{13 / 2}}\left[90(x-1)^{4}+435(x-1)^{3}\right. \\
+ & \left.700(x-1)^{2}+434(x-1)+77\right] \\
> & 0
\end{aligned}
$$

for $\mathrm{x}>1$. This implies that $f^{\prime}(x)>0$ and that $f(x)$ is increasing on $(1, \infty)$.

By the asymptotic expansion

$$
\psi(x) \sim \ln x-\frac{1}{2 x}-\frac{1}{12 x^{2}}+\frac{1}{120 x^{4}}-\frac{1}{252 x^{6}}+\cdots
$$

as $\mathrm{x} \rightarrow \infty$ in [[1], p. 259] and the well-known formula

$$
\psi(x+1)=\psi(x)+\frac{1}{x},
$$

we easily find

$$
\psi(x+1) \sim \ln x+\frac{1}{2 x}-\frac{1}{12 x^{2}}+\frac{1}{120 x^{4}}-\frac{1}{252 x^{6}}+\cdots
$$

as $\mathrm{x} \rightarrow \infty$. Hence, it follows that

$$
\begin{aligned}
& f(n)=\psi(n+1)-\ln n-\ln \cosh \frac{1}{\sqrt{n}}+\gamma \\
& =\frac{1}{2 n}+O\left(\frac{1}{n}\right)-\ln \frac{e^{-1 / \sqrt{n}}+e^{1 / \sqrt{n}}}{2}+\gamma \\
& \rightarrow \gamma, n \rightarrow \infty .
\end{aligned}
$$

Taking into account that $f(x)$ is increasing on $(1, \infty)$ reveals

$$
f(1) \leq f(n)<\lim _{n \rightarrow \infty} f(n)=\gamma, n \in \mathbb{N} .
$$

Combining (1), (3.1), and (3.2) concludes that the double inequality (1.1) holds for all $\mathrm{n} \geq 1$ and that the bounds $\alpha=0.5662 \ldots$ and $\beta=\gamma=0.5772 \ldots$ in (1.1) are the best impossible. The proof of Theorem 1.1 is complete.

\section{References}

[1] M. Abramowitz and I. A. Stegun (Eds), Handbook of Mathematical Functions with Formulas, Graphs, and Mathematical Tables, National Bureau of Standards, Applied Mathematics Series 55, 10th printing, Washington, 1972.

[2] H. Alzer, Inequalities for the harmonic numbers, Math. Z. 267 (2011), no. 1-2, 367-384.

[3] H. Alzer, On some inequalities for the gamma and psi functions, Math. Comp. 66 (1997), no. 217, 373-389.

[4] H. Alzer, Sharp inequalities for the harmonic numbers, Expo. Math. 24 (2006), no. 4, 385-388.

[5] N. Batir, Some new inequalities for gamma and polygamma functions, J. Inequal. Pure Appl. Math. 6 (2005), no. 4, Art. 103.

[6] C.-P. Chen, Inequalities for the Euler-Mascheroni costant, Appl. Math. Lett. 23 (2010), no. 2, 161-164.

[7] C.-P. Chen, Sharpness of Negoi's inequality for the EulerMascheroni constant, Bull. Math. Anal. Appl. 3 (2011), no. 1, 134-141.

[8] C.-P. Chen and C. Mortici, New sequence converging towards the Euler-Mascheroni constant, Comp. Math. Appl. 64 (2012), no. 2, 391-398.

[9] D. W. DeTemple, A quicker convergence to Euler's constant, Amer. Math. Monthly 100 (1993), no. 5, 468-470.

[10] B.-N. Guo and F. Qi, Two new proofs of the complete monotonicity of a function involving the psi function, Bull. Korean Math. Soc. 47 (2010), no. 1, 103-111.

[11] B.-N. Guo and F. Qi, Sharp bounds for harmonic numbers, Appl. Math. Comput. 218 (2011), no. 3, 991-995.

[12] B.-N. Guo and F. Qi, Sharp inequalities for the psi function and harmonic numbers, Analysis (Berlin) 34 (2014), no. 2, 201-208. 
[13] E. A. Karatsuba, On the computation of the Euler constant, Numer. Algor. 24 (2000), no. 1-2, 83-97.

[14] W.-H. Li, F. Qi, and B.-N. Guo, On proofs for monotonicity of a function involving the psi and exponential functions, Analysis (Munich) 33 (2013), no. 1, 45-50.

[15] C. Mortici, A quicker convergence toward the constant with the logarithm term involving the constant e, Carpathian J. Math. 26 (2010), no. 1, 86-91.

[16] C. Mortici, Improved convergence towards generalized EulerMascheroni constant, Appl. Math. Comput. 215 (2010), no. 9, 3443-3448.

[17] C. Mortici, New approximations of the gamma function in terms of the digamma function, Appl. Math. Lett. 59 (2010), no. 1, 97100 .

[18] C. Mortici, On new sequences converging towards the EulerMascheroni constant, Comput. Math. Appl. 59 (2010), no. 8, 2610-2614.

[19] T. Negoi, A faster convergence to Euler's constant, Math. Gaz. 83 (1999), no. 498, 487-489.
[20] P. Paule and C. Schneider, Computer proofs of a new family of harmonic number identities, Adv. Appl. Math. 31 (2003), no. 2, 359-378.

[21] F. Qi, Complete monotonicity of functions involving the qtrigamma and q-tetragamma functions, Rev. R. Acad. Cienc. Exactas Fís. Nat. Ser. A Math. RACSAM. 109 (2015), in press.

[22] F. Qi, R.-Q. Cui, C.-P. Chen and B.-N. Guo, Some completely monotonic functions involving polygamma functions and an application, J. Math. Anal. Appl. 310 (2005), no. 1, 303-308.

[23] F. Qi and Q.-M. Luo, Complete monotonicity of a function involving the gamma function and applications, Period. Math. Hungar. 69 (2014), no. 2, 159-169.

[24] A. Sîntămărian A generalization of Euler's constant, Numer. Algor. 46 (2007) no. 2, 141-151.

[25] M. B. Villarino, Ramanujan's harmonic number expansion into negative powers of a triangular num- ber, J. Inequal. Pure Appl. Math. 9 (2008), no. 3, Art. 89.

[26] R. M. Young, Euler's constant, Math. Gaz. 75 (1991), 187-190. 